\title{
Étude en laboratoire du frottement \\ dans les matériaux granulaires
}

\section{S. KHATI}

I. SHAHROUR

Laboratoire de Mécanique de Lille (URA 1441),

École centrale de Lille, 59651 Villeneuve-d'Ascq

Cedex

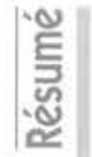

Dans cet article, on présente une étude expérimentale du frottement dans les matériaux granulaires. Elle est basée sur des essais réalisés à la boite de cisaillement direct en utilisant trois matériaux (silice, calcite et quartz) et différents modes de contact: bloc-bloc, bloc-grains et grains-grains. L'analyse des résultats des essais permet d'étudier l'effet de la rugosité et la forme des grains sur le frottement de contact. La contribution de l'effet de structure dans le frottement mobilisé est analysée par une confrontation des résultats de cisaillement sablesable aux essais de cisaillement bloc-bloc et sable-bloc.

\section{Experimental study of friction in granular materials}

This paper includes an experimental study of friction in granular materials. It is based upon direct shear tests performed on three materials (silica, calcite, quartz) with various contact conditions: block-block, block-grains and grains-grains. Analysis of experimental results permits to investigate the influence of both roughness and shape of grains on friction resulting from contact. The contribution of the structure effect is analysed by comparison of results of conventional shear test performed on sand with tests performed on block-block or sand-block. 


\section{1}

\section{Introduction}

L'optimisation des installations de stockage et de transport des matériaux en grains (silos, trémies, transporteuses à vis ou à bande, transport pneumatique...) utilisées dans les industries chimiques, pétrolières, pharmaceutiques et agroalimentaires requiert une bonne modélisation du comportement complexe de ces matériaux.

Le frottement constitue le paramètre fondamental caractérisant le comportement des matériaux granulaires. Il peut être déterminé à partír des essais de laboratoire ou à partir des modèles macroscopiques utilisant l'angle de frottement de contact comme paramètre de base, Les techniques expérimentales de détermination du frottement de contact sont basées essentiellement sur le glissement de deux grains ou bloc de grains avec ou sans traitement de surface (Penman, 1953; Procter et al., 1974; Nascimento, 1977; Ishibashi et al., 1994) ou sur le cisaillement d'un ensemble de grains sur un bloc de grains enchâssés (Tschebotarioff et Welch, 1948; Rowe, 1962; Horne, 1965; Skinner, 1969; Lambe et Whitman, 1969), Dans ce second cas, les essais mettent en jeu l'influence des caractéristiques pétrographiques ou morphométriques des grains sur leurs propriétés mécaniques,

Les résultats des essais de mesure du frottement des milieux granulaires comportent généralement de fortes dispersions. Une part de ces dispersions est liée à la présence d'humidité (Horn et Deere, 1962), à la rugosité et au traitement chimique des surfaces (Mitchell, 1993). Elles sont plus importantes dans le cas du glissement grain sur grain dues essentiellement à la microtopologie des surfaces de contact (Procter et Barton,
1974). Les dispersions des mesures et la divergence des procédés utilisés rendent l'interprétation et la comparaison des résultats publiés très difficiles.

La relation entre l'angle de frottement de contact et l'angle de frottement apparent dans un matériau granulaire est très complexe. Elle fait intervenir de nombreux paramètres liés à la morphologie des grains, leurs rugosité et assemblage. Dans cet article, on propose d'étudier le frottement au sein des matériaux granulaires à l'aide de la boîte de cisaillement de Casagrande. En utilisant différentes procédures expérimentales, on cherche à analyser les contributions de principaux facteurs dans la mobilisation du frottement intergranulaire.

\section{2}

\section{Dispositif expérimental et mode opératoire}

\section{1}

\section{Dispositif expérimental}

Le dispositif expérimental utilisé a été développé au laboratoire des matériaux granulaires de l'École des mines de Douai autour de la boite de cisaillement de Casagrande (Fig. 1) (Khati, 1996). Il est équipé de (i) deux capteurs de déplacement LVDT pour la mesure des variations de la hauteur des échantillons; leur disposition, symétriques par rapport à l'axe de transmission de la charge, permet de mesurer l'inclinaison du chapeau au cours du cisaillement; (ii) un capteur LVDT pour la mesure du déplacement relatif tangentiel; (iii) un capteur de force à jauge pour la mesure de l'effort

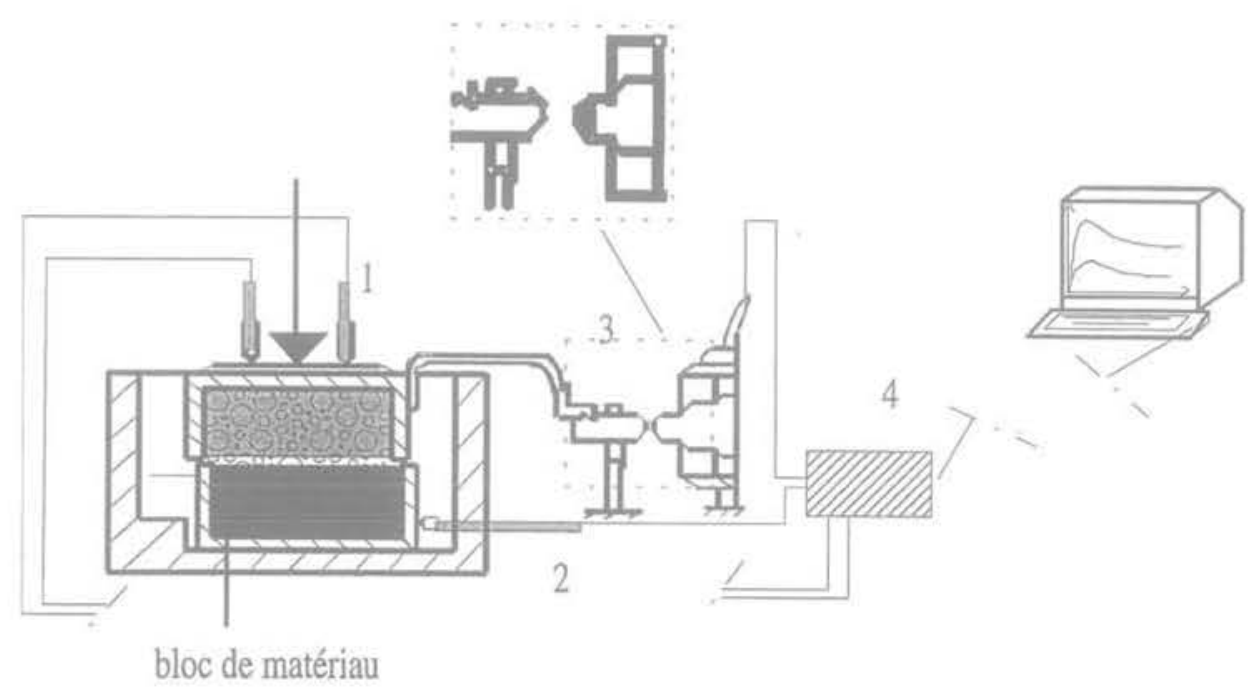

\author{
1 : Capteurs de déplacement vertical \\ 3: Capteur de force
}

2: Capteur de déplacement horizontal

4: Système d'acquisition 
TABLEAUI Minéralogie des matériaux utilisés.

Mineralogy of used materials.

\begin{tabular}{l|c|c|c|c|c|c} 
Échantillon & $\mathrm{CO} \mathrm{Ca}(\%)$ & $\mathrm{SiO}_{2}(\%)$ & $\mathrm{Al}_{2} \mathrm{O}_{3}(\%)$ & $\mathrm{Fer}(\%)$ & $\mathrm{K}_{2} \mathrm{O}(\%)$ & $\mathrm{CaO}(\%)$ \\
\hline Calcite & 91 & 5 & 0,7 & 2,7 & 0,2 & - \\
\hline Silice & - & 77 & 2 & 5 & 1 & 12 \\
\hline Quartz & - & 80 & 10 & 2 & 7 & 1 \\
\hline
\end{tabular}

tangentiel et (iv) un système d'acquisition automatisé piloté par micro-ordinateur. Le capteur de force est mis en contact par une liaison simple avec la partie supérieure de la boîte de cisaillement. Les capteurs de déplacement et de forces présentent des erreurs relatives de mesure de $1 \mu \mathrm{m}$ pour une course totale de $10 \mathrm{~mm}$ et de $0,5 \mathrm{~N}$ pour une charge totale de 500 daN respectivement.

\section{2}

\section{Mode opératoire}

Les essais ont été réalisés sur des échantillons cohérents (en bloc) ou en grains (sable). Les échantillons en bloc sont directement découpés dans la roche aux dimensions de la boîte de cisaillement. Le bloc de la partie supérieure a une dimension longitudinale inférieure de la longueur relative totale du déplacement par rapport à la partie inférieure. Ils sont lavés et étuvés à $105^{\circ} \mathrm{C}$ pendant 24 heures, puis laissés à l'air libre pendant la durée des essais. Ce procédé permet d'éviter la contamination de la surface de cisaillement par collage ou cimentation de grains (ciment, plâtre, résine, etc.).

Nous avons utilisé trois procédures liées aux mouvements des grains au niveau du plan de cisaillement (Fig. 2). Chaque procédure correspond à un type d'essais, à savoir bloc-bloc, sable-bloc et sable-sable. Dans le premier cas (bloc-bloc), la structure de l'échantillon est rigide (pas de mouvements relatifs entre les grains). Il permet le contact et le frottement entre les éléments composant les blocs par glissement. Dans le cas sablebloc, la partie inférieure est constituée d'un bloc intact et la partie supérieure de grains de même nature. Et enfin, le cas sable-sable représente les essais classiques de cisaillement direct où le plan de cisaillement est localisé dans la zone de séparation des deux demi-boîtes.

Les essais ont été réalisés à des confinements relativement faibles, pour minimiser la cassure des grains (Wilkins, 1970), à une vitesse de $0,4 \mathrm{~mm} / \mathrm{mn}$ avec un enregistrement moyen de 20 points à la minute.
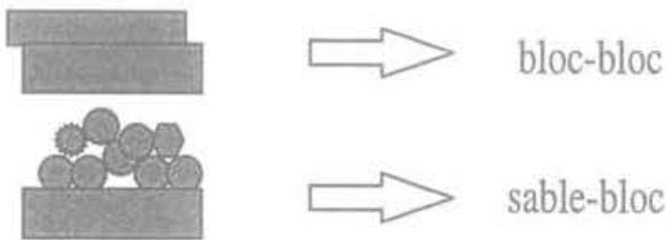

sable-bloc
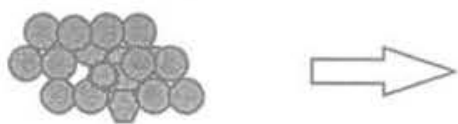

sable-sable

FIG. 2 Illustration des procédures expérimentales.

Illustration of experimental procedures.

\section{3}

\section{Matériaux utilisés}

Les matériaux utilisés sont de trois natures différentes et désignés par leur composante majoritaire. Leur minéralogie, présentée dans le tableau I, a été déterminée par analyse semi-quantitative par fluorescence et diffraction aux rayons-X et par observation au microscope électronique (MEB et MET). L'échantillon dit de «silice ») est composé essentiellement de silice " amorphe » de type opale CT. Cassé, il donne lieu à des éclats de roche acérés et fins. La cimentation est de même nature que la majorité des éléments qui composent le matériau. Sa dureté est équivalente à 7 sur l'échelle de Mohs. L'échantillon dit de «quartz» est issu du grès des Vosges. Il est formé en majorité de grains de quartz faiblement cimentés, donnant lieu à une porosité relativement élevée (environ $38 \%$ ). Cassé, le matériau est réduit à des grains intacts de différentes tailles. Sa dureté est de 7. L'échantillon dit de «calcite » est formé de microsparites. Cassé, il donne lieu à des grains de forme ovoíde et allongée. Sa dureté est équivalente à 3 (plus facile à rayer que la silice et le quartz).

La rugosité des matériaux a été imposée par polissage des surfaces au carbure de silicium. Le choix de l'état de surface a été fait de façon à obtenir une surface suffisamment rugueuse ayant des variations de volume relativement faibles et une surface lisse, sans induire un frottement parasite (Harr, 1981). La rugosité des surfaces, notion relative, est présentée par la grosseur du grain équivalent. Ainsi, la surface lisse correspond à une grosseur de grain de $12,5 \mu \mathrm{m}$ de diamètre et la surface rugueuse à une grosseur de $180 \mu \mathrm{m}$. Des mesures de contrôle au rugosimètre (Sander, 1992) ont été effectuées pour constater sa répartition et son homogénéité. Les valeurs moyennes de $\mathrm{R}$ obtenues pour les échantillons de silice et de calcite sont de $38,5 \mu \mathrm{m}$ et $2,6 \mu \mathrm{m}$ pour les surfaces rugueuses et lisses respectivement. Nous n'avons pas pu aller au bout de la mesure pour les échantillons de quartz à cause de sa forte porosité.

Les courbes de granulométrie (Fig. 3) constituẻes par mélange des différentes fractions obtenues par concassage et tamisat des échantillons en bloc sont semblables pour les trois matériaux. Le choix de l'uniformité du sable a été motivé par le besoin d'assurer un maximum de contact avec la surface du bloc dans le cas des essais sable-bloc. On notera que le diamètre inférieur des sables utilisés est compris entre les deux limites de rugosité définies.

Les résultats des mesures de la sphéricité (Riley, 1941) et de l'angularité (Wadell, 1933), réalisées à l'aide du traitement d'images (Khati, 1996), montrent des grains de quartz plus arrondis et plus anguleux. Les grains de silice sont plus allongés avec des arrêtes plus 


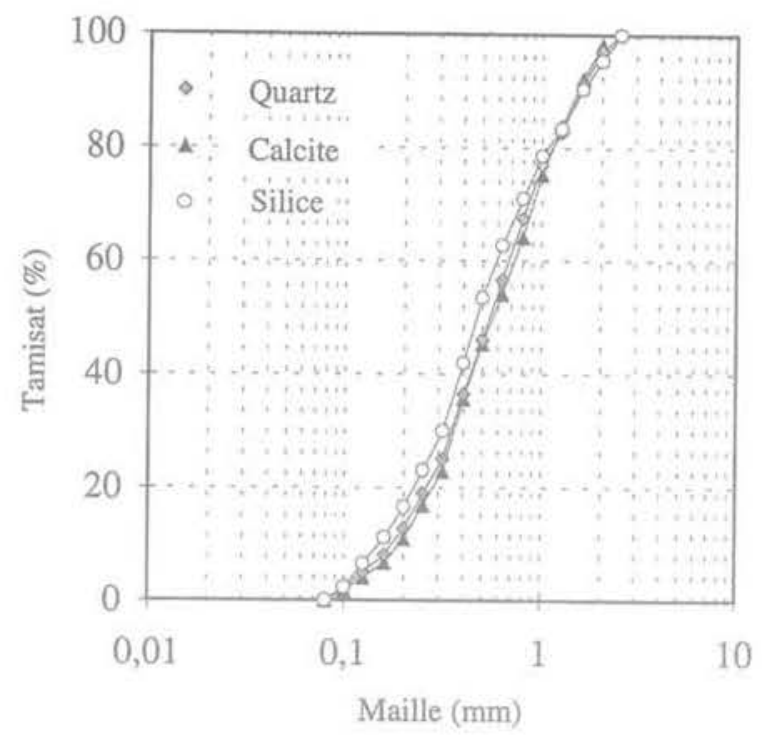

FG. 3

Courbes granulométriques des sables utilisés.

Grain size curves of used materials.

vives, contrairement aux grains de calcite qui présentent une forme allongée à arrêtes arrondies (Fig. 4). Au touché, le quartz est rugueux, et la silice est lisse. L'examen à la loupe de la surface confirme les résultats obtenus au touché et au traitement d'images. Malgré leur différence de nature, les grains de quartz et de calcite présentent des paramètres morphométriques (sphéricité, angularité) peu différents, ce qui leur confèrent des caractéristiques de mise en place voisines [1 (quartz) $=I_{e}$ (calcite)] (tableau II). L'augmentation de l'écart entre les indices des vides limites des échantillons de silice peut être attribuée à la diminution de la sphéricité.

\section{Résultats expérimentaux}

\section{1}

\section{Mesure du frottement}

La figure 5 représente un exemple de réponse des matériaux en fonction du déplacement tangentiel relatif pour les différentes procédures, à savoir bloc-bloc (bb), sable-bloc (s-b) et sable (s-s). Dans le cas des essais bloc-bloc, on observe une réponse de type élastiqueparfaitement plastique pour les deux états de surface (lisse et rugueux), à l'exception des essais de silice à surface rugueuse où on note un léger pic. Dans le cas des essais sable-bloc, on observe un comportement sans pic avec une augmentation linéaire du frottement mobilisé en fonction du déplacement pour les diffé-

TABLEAU II Caractéristiques des sables utilisés. Properties of used sands.

\begin{tabular}{|c|c|c|c|}
\hline Matériau & Silice & Quartz & Calcite \\
\hline $\mathrm{Cu}=\mathrm{d}_{10} / \mathrm{d}_{10}$ & 3,89 & 3,88 & 3,82 \\
\hline $\mathrm{d}_{\text {sio }}(\mathrm{mm})$ & 0,470 & 0,548 & 0,568 \\
\hline$e_{\max }$ & 1,093 & 0,755 & 0,789 \\
\hline $\mathrm{e}_{\min }$ & 0,684 & 0,436 & 0,483 \\
\hline $\mathrm{I}_{\mathrm{v}}=\mathrm{e}_{\max }-\mathrm{e}_{\min }$ & 0,409 & 0,319 & 0,306 \\
\hline Sphéricité & 0,777 & 0,901 & 0,809 \\
\hline Angularitê (c) & 0,343 & 0,152 & 0,254 \\
\hline Dureté & 7 & 7 & 3 \\
\hline
\end{tabular}
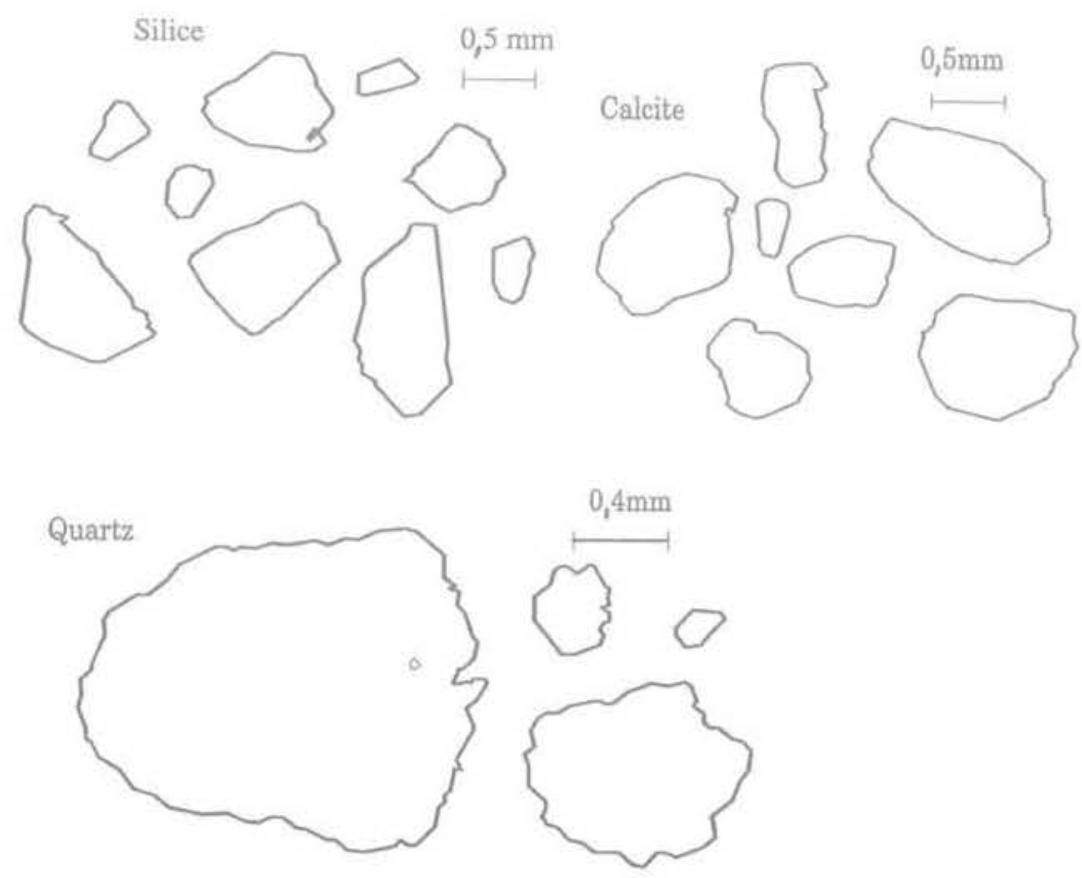

FG.4 Analyse morphométrique des matériaux en grains obtenue par traitement d'images.

Morphometric analysis of grains using image processing. 

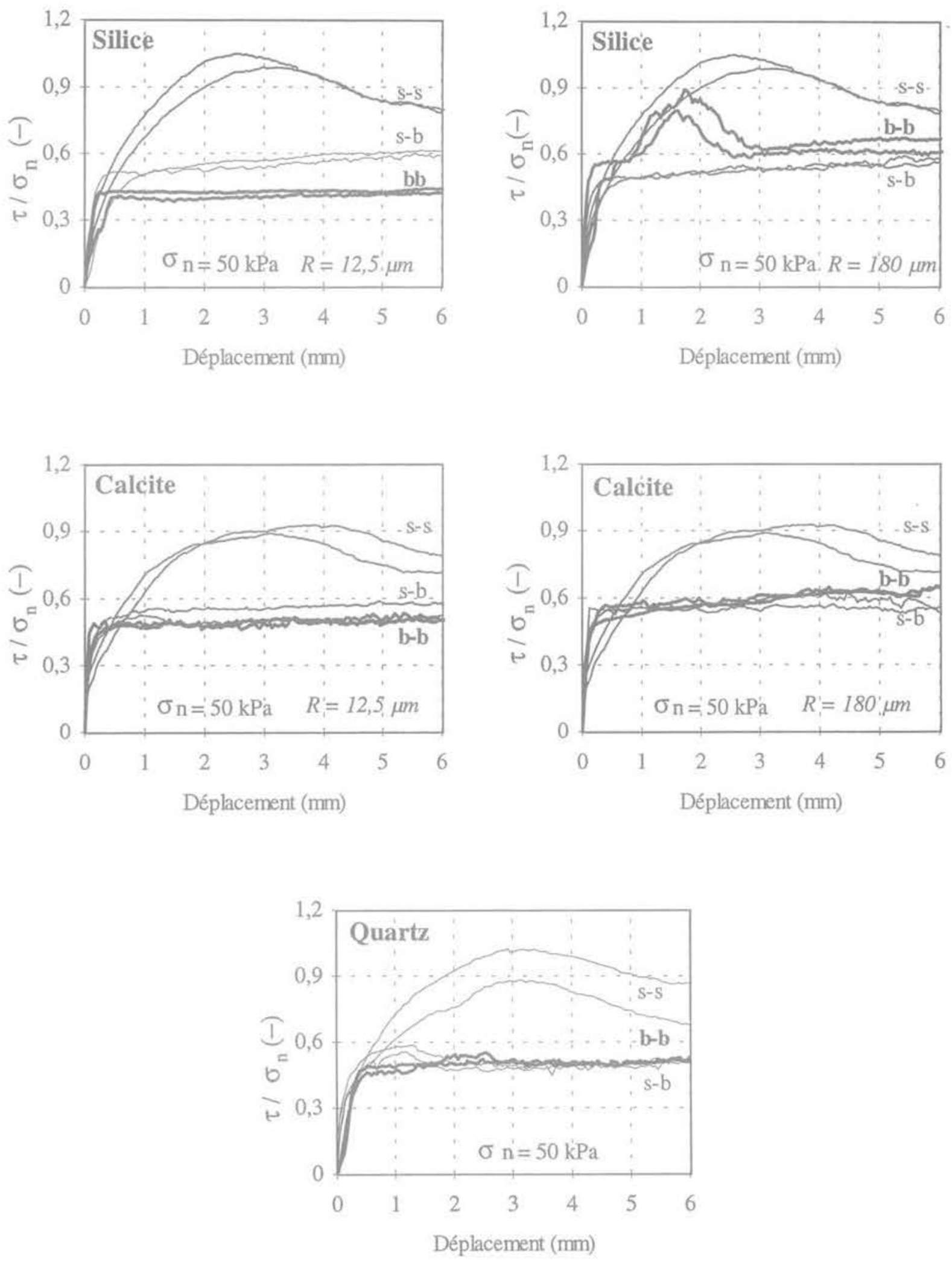

FG.5 Résultats des essais obtenus avec les différentes procédures $\left(\sigma_{\mathrm{n}}=50 \mathrm{kPa}\right)$. Résults obtained with proposed procedures $\left(\sigma_{\mathrm{n}}=50 \mathrm{kPa}\right)$.

rents échantillons. L'état de surface n'a pratiquement pas d'influence sur la réponse globale. Les essais du quartz présentent un comportement du type sable dense avec un pic prononcé aux faibles confinements.

L'évolution du coefficient de variation, déterminé sur une dizaine d'essais soumis aux mêmes conditions de chargement, est illustrée dans les figures $6 a$ et $6 \mathrm{~b}$. On note une importante dispersion des mesures au début du cisaillement, mais au-delà de $1 \mathrm{~mm}$ de déplacement, les variations se stabilisent et restent voisines d'une valeur moyenne de l'ordre de $5 \%$ pour les surfaces lisses et de $7 \%$ pour les surfaces rugueuses. 

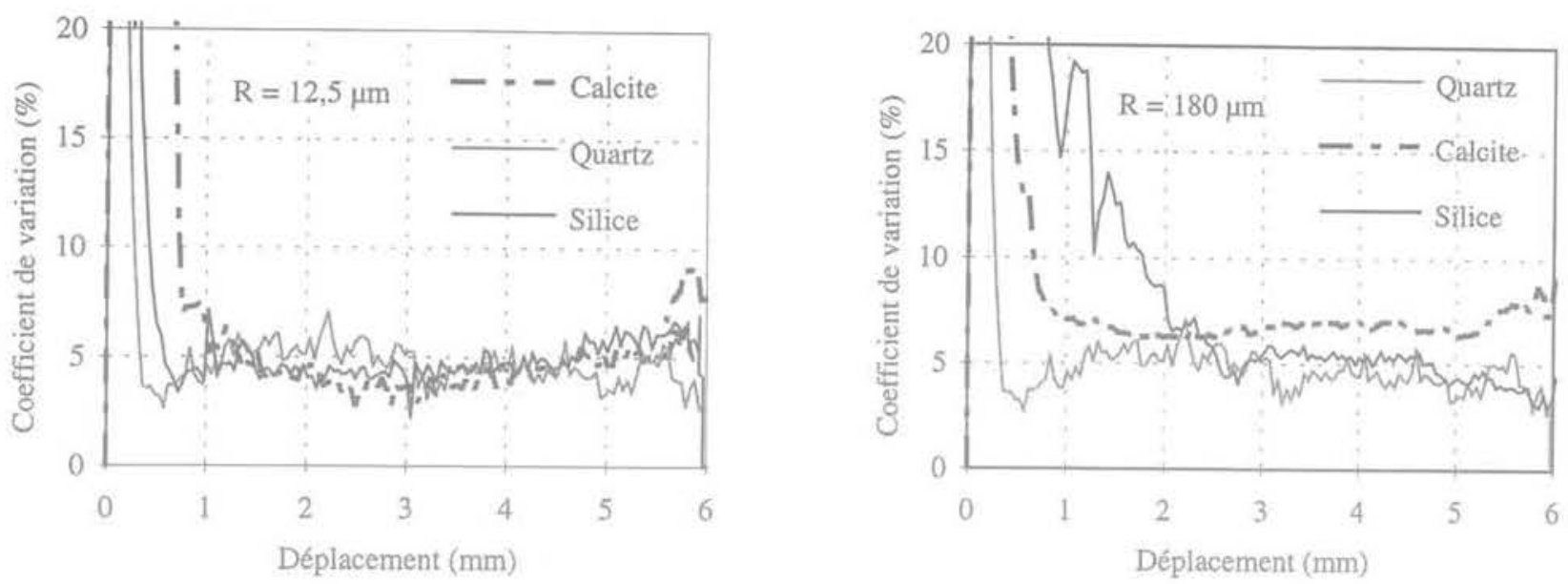

FG. 6 Analyse des dispersions de mesure. a: surface lisse, b: surface rugueuse. Analysis of the experimental dispersion. a: smooth surface, b: rough surface.

Compte tenu de cette faible dispersion, l'angle de frottement sera déterminé à partir de la valeur moyenne des cisaillements mesurés au palier.

\section{2}

\section{Présentation et discussion des résultats}

\subsection{3}

\section{Essais bloc-bloc}

Les essais de cisaillement bloc-bloc ont été réalisés avec les trois matériaux pour différents niveaux de confinement (de 10 à $250 \mathrm{kPa}$ ) sur des échantillons à surfaces lisses $(R=12,5 \mu \mathrm{m})$ ou rugueuses $(R=180 \mu \mathrm{m})$.
Les résultats obtenus sont illustrés dans la figure 7. On peut constater que dans le cas des surfaces lisses, les angles de frottement mesurés sont peu dépendants du confinement. L'angle de frottement moyen obtenu avec l'échantillon de calcite $\left(26^{\circ}\right)$ est plus élevé que ceux obtenus avec les échantillons de silice $\left(23^{\circ}\right)$ et de quartz $\left(24,5^{\circ}\right)$. L'augmentation de la rugosité de surface se manifeste par un accroissement de l'angle de frottement en particulier pour les matériaux à dureté élevée (de $10,5^{\circ}$ pour la silice mais seulement de $4^{\circ}$ pour la calcite). L'examen des surfaces rugueuses montre des épontes plus vives pour les échantillons de silice et plus arrondies pour la calcite. Par ailleurs, nous avons noté la présence d'une fine pellicule de matériau, à la fin des essais des échantillons de calcite, liées à I'usure des épontes avec l'augmentation de la contrainte normale.

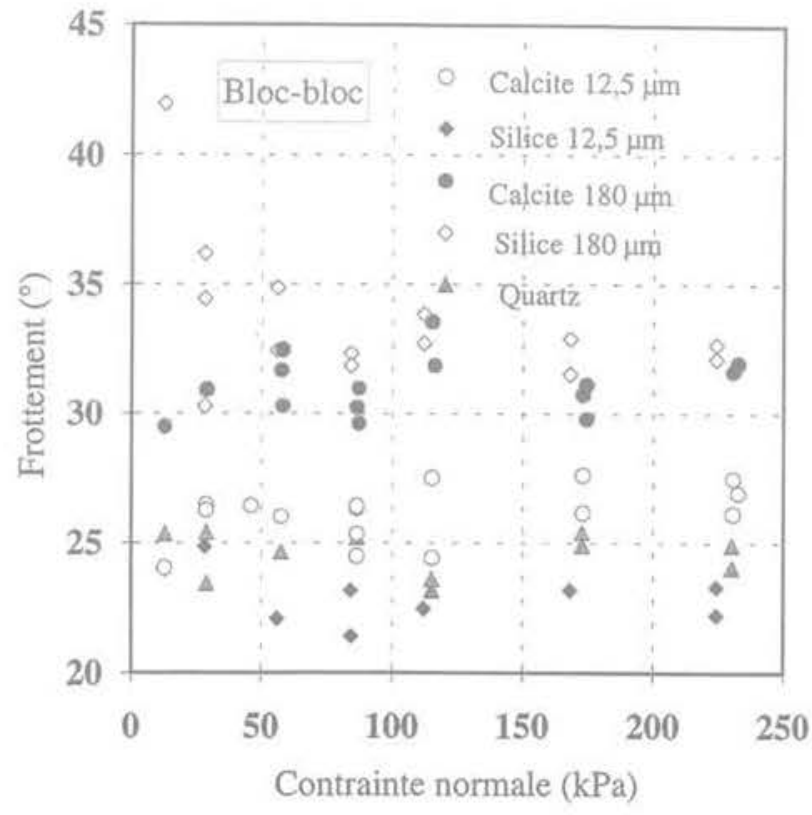

FIG.7 Frottement en fonction de la contrainte normale : essais bloc-bloc.

Friction in terms of normal stress : tests blockblock.

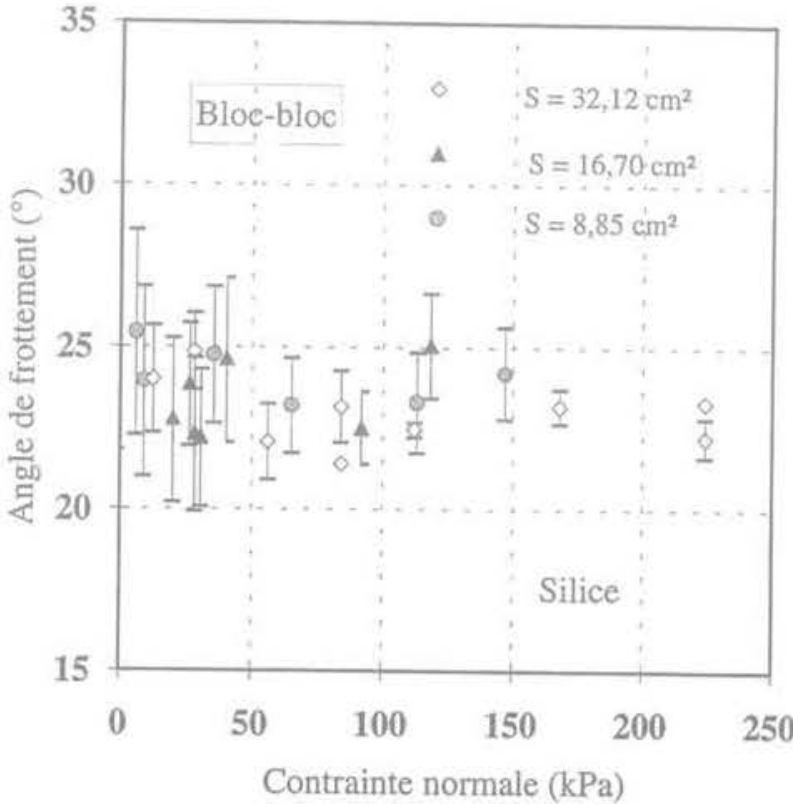

FiG.8 Influence sur le frottement des dimensions des surfaces de glissement. Influence of the surface dimension on friction. 
Des essais ont été également effectués pour étudier l'influence des dimensions des blocs sur l'angle de frottement. Les résultats obtenus avec des blocs de silice sont donnés dans la figure 8. On constate que l'angle de frottement moyen est peu influencé par les dimensions de la surface de bloc. En ce qui concerne les fluctuations, on note qu'elles s'amplifient avec la réduction des dimensions des blocs, en particulier aux faibles niveaux de contraintes.

\section{2}

\section{Essais sable-bloc}

Les essais de cisaillement sable-bloc ont été réalisés avec les trois matériaux sur des blocs à surfaces lisses ou rugueuses et différents nivaux de confinement. Les résultats obtenus sont donnés dans la figure 9 . On constate que les angles de frottement obtenus sont peu influencés par la rugosité de la surface des blocs. Avec les blocs de calcite, on obtient un angle de frottement moyen de $28,5^{\circ}$ qui est proche de la valeur moyenne des angles obtenus avec les essais bloc-bloc à surfaces lisses et à surfaces rugueuses. Avec les blocs de silice, on obtient un angle de frottement de $30^{\circ}$ qui est proche de l'angle de frottement obtenu avec les essais bloc-bloc à surfaces rugueuses $\left(33^{\circ}\right)$. Avec les blocs de quartz, on obtient un angle de frottement de $27^{\circ}$ qui est légèrement supérieur à l'angle obtenu avec les essais bloc-bloc $\left(25^{\circ}\right)$. Ces résultats montrent que dans le cas d'une surface lisse, l'angle de frottement grain-bloc est supérieur à celui obtenu avec les essais bloc-bloc, en particulier pour les grains de silice. Ce résultat peut s'expliquer par un accroissement de la rugosité de la surface de contact induit par le contact grains-bloc, en particulier pour les grains à dureté élevée et à vives arrêtes comme ceux de la silice. Avec les surfaces rugueuses, on note une légère diminution de l'angle de frottement qui peut s'expliquer par une réduction de la rugosité de contact grain-bloc par rapport à la rugosité de contact bloc-bloc à surfaces rugueuses.

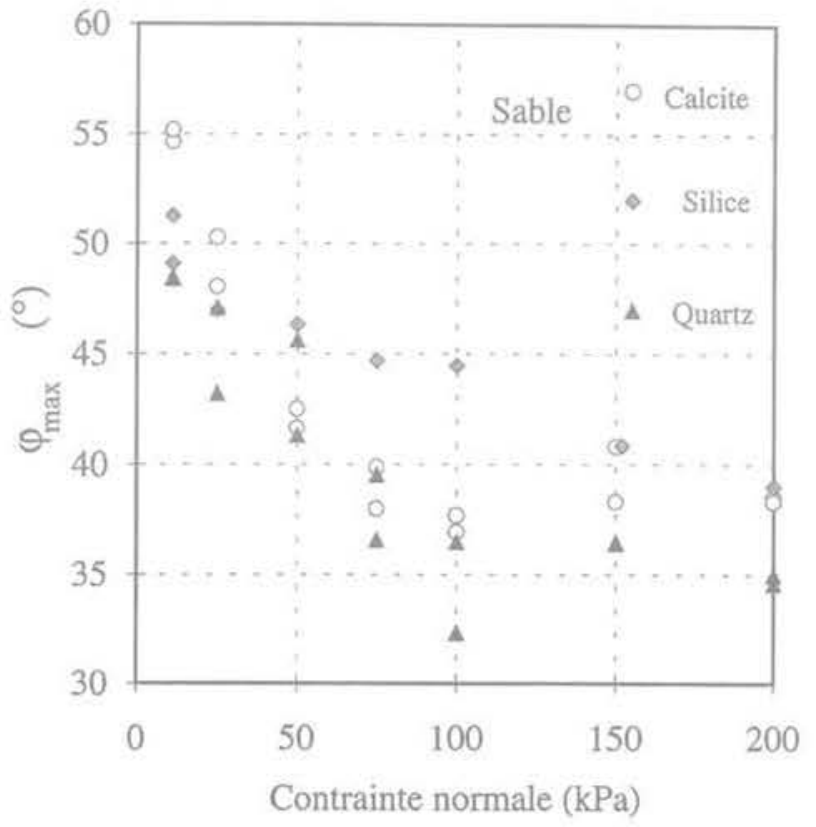

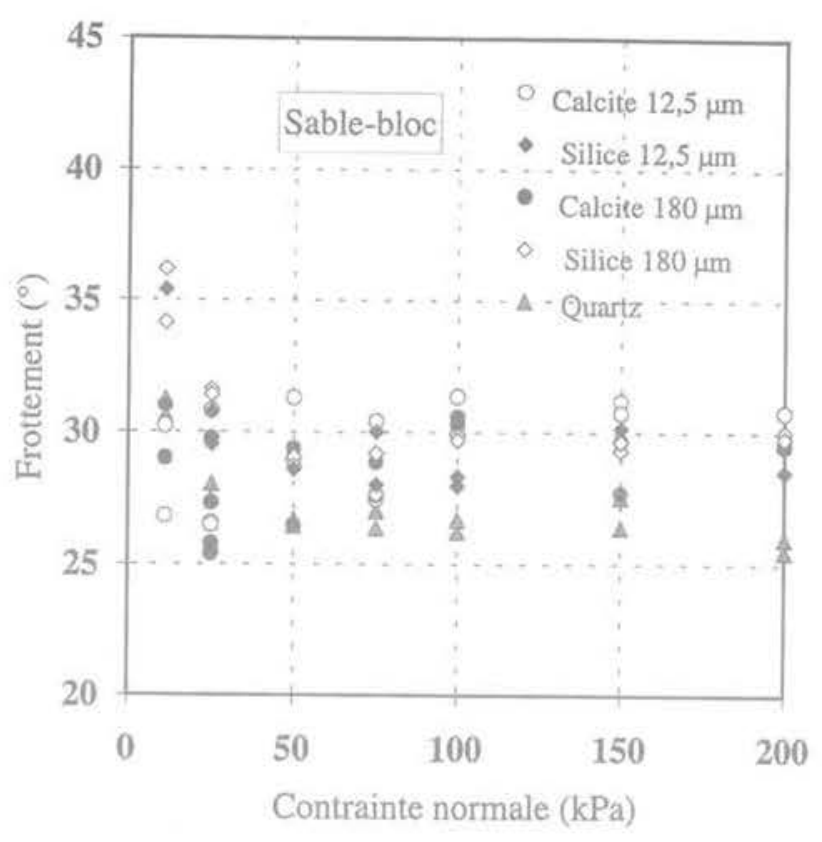

คG 9 Frottement en fonction de la contrainte normale : essais sable-bloc.

Friction in terms of normal stress; tests blocksand.

\section{A, 93}

\section{Essais de cisaillement sable-sable}

Dans le cas des essais de cisaillement sable-sable, on note la présence d'un pic suivi par un radoucissement puis par un palier. Du fait que le frottement au pic est associé à des mécanismes de localisation, ses valeurs sont données et commentées à titre indicatif. La figure 10 montre l'évolution de l'angle de frottement au pic $\left(\varphi_{\max }\right)$ avec le niveau de contrainte pour les trois

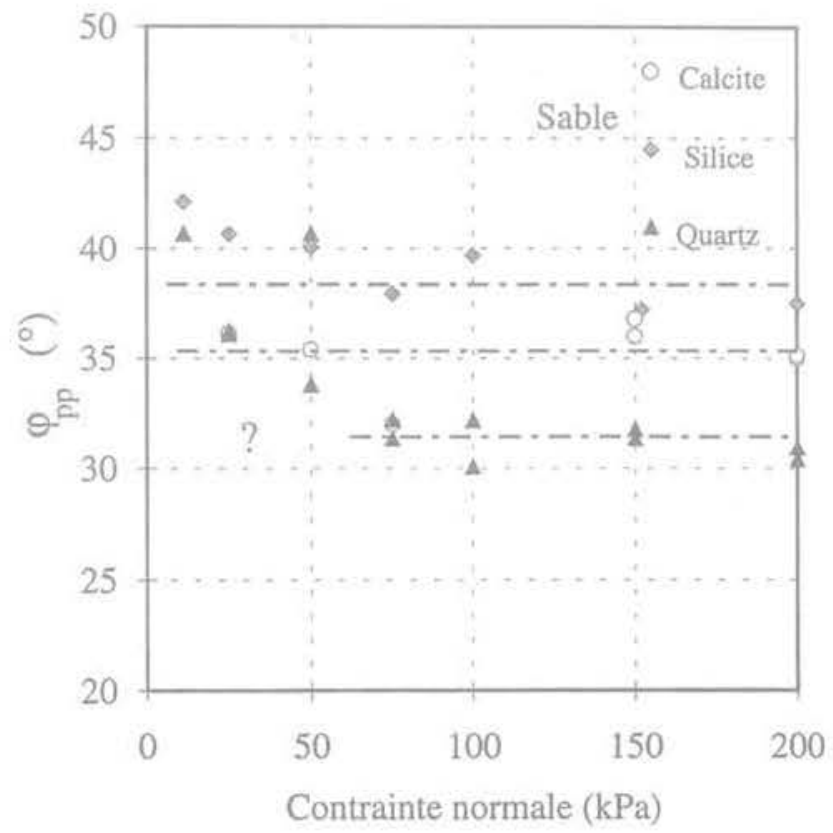

fiG. 10 Frottement en fonction de la contrainte normale: essais sable-sable. a : angle au pic, b: angle au palier.

Friction in terms of normal stress : tests sand-sand. a: peak angle, b: limit angle. 
TABLEAUIII Valeur de l'angle de frottement moyen pour les différentes procédures. Values of friction angle determined from proposed procedures.

\begin{tabular}{|c|c|c|c|c|c|c|}
\hline \multicolumn{7}{|c|}{ Frottement $\left({ }^{\circ}\right)$} \\
\hline Procedures & \multicolumn{2}{|c|}{ Bloc-bloc (b-b) } & \multicolumn{2}{|c|}{ Sable-bloc (s-b) } & \multicolumn{2}{|c|}{ Sable } \\
\hline $\begin{array}{l}\text { Etat de } \\
\text { surface }\end{array}$ & $\begin{array}{c}\text { Lisse } \\
(12,5 \mu \mathrm{m})\end{array}$ & $\begin{array}{l}\text { Rugueux } \\
(180 \mu \mathrm{m})\end{array}$ & $\begin{array}{c}\text { Lisse } \\
(12,5 \mu \mathrm{m})\end{array}$ & $\begin{array}{l}\text { Rugueux } \\
(180 \mu \mathrm{m})\end{array}$ & $\varphi_{\max }$ & $\varphi_{p p}$ \\
\hline Calcite & $26,1 \pm 1,5$ & $30,2 \pm 1,9$ & $28,6 \pm 1,2$ & $29,5 \pm 1,5$ & $42,9 \pm 6,4$ & $35,2 \pm 1,5$ \\
\hline Silice & $22,9 \pm 0,9$ & $33,6 \pm 3,3$ & $30,5 \pm 1,5$ & $29,7 \pm 1,5$ & $46,2 \pm 4,1$ & $39,3=1,8$ \\
\hline Quartz. & \multicolumn{2}{|c|}{$24,7 \pm 1,2$} & \multicolumn{2}{|c|}{$27,3 \pm 1,2$} & $40,1 \pm 5,5$ & $32,8 \pm 3,2$ \\
\hline
\end{tabular}

matériaux. On constate que les valeurs de cet angle sont èlevées, en particulier aux faibles niveaux de confinement. Dans le cas des grains de calcite, I'angle au pic passe de $55^{\circ}$ à $38^{\circ}$, lorsque la contrainte normale croit de 10 à $200 \mathrm{kPa}$. Cette variation est plus modérée dans le cas des grains de silice (resp. de quartz): l'angle au pic décroit de $50^{\circ}$ (resp. $48^{\circ}$ ) à $40^{\circ}$ (resp. $35^{\circ}$ ) lorsque la contrainte normale croit de 10 à $200 \mathrm{kPa}$.

En ce qui concerne l'angle de frottement au palier $(\varphi)$, on note une légère dépendance de cet angle par rapport à la contrainte normale dans le cas des grains de calcite et de silice: les angles de frottement moyens mesurés avec ces matériaux sont respectivement de $35^{\circ}$ et $39^{\circ}$. Avec les grains de quartz, on note que la contrainte normale affecte la valeur de l'angle de frottement, en particulier pour les faibles confinements, qui décroit de $40^{\circ}$ à $30^{\circ}$ lorsque la contrainte normale croit de 10 à $100 \mathrm{kPa}$.

Ces résultats montrent que les angles de frottement mesurés sont influencés par la morphologie des particules. En effet, les grains de quartz et de silice, composés en majorité du même minéral, présentent des comportements différents au pic et au palier. La présence d'une forte angularité associée à une faible sphéricité peut procurer plus de stabilité entre les grains empilés, ce qui nécessiterait un effort relatif plus important lors du cisaillement pour les échantillons de silice (Biarez et al., 1989),

Une comparaison des angles de frottement mesurés avec les trois procédures (tableau III) montre que le frottement des matériaux en grains (essais sable-sable) est plus élevé que ceux obtenus avec les essais sablebloc ou bloc-bloc à surfaces lisses ou rugueuses. Au niveau du frottement de contact des grains obtenus, on s'attend dans le cas des essais sable-sable à un angle de frottement de contact proche de celui mesuré avec les essais sable-bloc. L'écart entre les angles de frottement apparent et de contact $\left(6^{\circ}\right.$ pour la calcite, $9^{\circ}$ pour la silice et $6^{\circ}$ pour le quartz) peut être attribué à un effet de structure faisant intervenir de nouveaux facteurs telles que les données morphométriques (rugosité des grains, angularité, sphéricité...) et la géométrie de l'assemblage. Ces facteurs ont une influence directe sur les possibilités de mouvements relatifs des grains (glissement et roulement) et de ruptures locales des grains.

\section{5}

\section{Conclusion}

Les essais de cisaillement réalisés sous différentes valeurs de la contrainte normale montrent que le frottement mesuré est fonction de la procédure expérimentale. Dans le cas du cisaillement bloc-bloc, le comportement des matériaux est peu sensible à la variation du confinement et aux dimensions de la surface de cisaillement. L'augmentation de la rugosité de surface induit un accroissement du frottement en particulier pour les matériaux à dureté élevée et à vives arrètes comme les grains de silice. Les essais de cisaillement sable-bloc donnent un angle de frottement moyen globalement indépendant de l'état de surface. Il se situe entre les angles de frottement bloc-bloc mesurés respectivement avec des surfaces lisses et rugueuses. Dans notre cas, les essais de cisaillement sable-sable donnent des angles de frottement au palier plus élevés que ceux mesurés avec les essais bloc-bloc et sable-bloc. Ceci peut être expliqué par un effet de structure de l'assemblage de grains qui peut induire au cours du cisaillement une réorganisation de l'assemblage et une dilatance dont la mobilisation requiert un supplément d'effort augmentant ainsi Yangle de frottement mobilisé. 
Biarez J., Favre J.-L., Hicher P.Y., Rahma A. - Correlations for granular media, classification logic and connections between classes. Powders and grains, Proc. ICMGM, Clermont-Ferrand. Biarez et Gourves éd.. p. 201-209, 1989

Harr M.E. - Mécanique des milieux formés de grains. Presses Polvtechniques Romandes, Lausanne, p. 514. 1981.

Horn H.M., Deere M.S. - Frictional characteristics of minerals. Geotechnique, 12 p. 319-335, 1962

Horne M.R. - The behaviour of an assembly of rotund, rigid, cohesionless particles: part 1 \& II Proc. Roy. Soc. serie A. vol. 286, p. 62.97, 1965.

Ishibashi 1. Perry III C. Agarwal T.K. Experimental determinations of contact friction for spherical glass particles. Soils and Foundations, JSSMFE, vol. 34 . $n^{\circ} 4$, p. $79-84,1994$.

Khati S. - Comportement des materiaux granulaires : étude micro-macro du frottement et de la dilatance. Thèse de doctorat, Université des Sciences et Technologies de Lille, p. 185, 1996.

Lambe T.W., Whitman R.V. - Soil mechanics. John Wiley \& Sons. New York. p. 553. 1969 .

Mitchell J.K. - Fundamentals of soil behavior. John WIley and Sons. New York. p. 426, 1993.

Nascimento U. - Goniometer for determining interparticule friction. 9th ICSMFE, Tokyo, $2^{6}$ ed., p. 229-233, 1977.

Penman A.D.M. - Shear characteristics of saturated silt measured in triaxial compression. Geotechnique, vol. 3, p. 312 328, 1953.

Procter D.C., Barton R.R. - Measurements of the angle of interparticle fiction. Geofechnique, vol.24, n० 4, p. 581-604, 1974. Riley N.A. - Projection sphericity. J. Sedim. Petrol., vol. 11, p. 94.97, 1941.
Rowe P.W. - The stress-dilatancy relation for static equilibrium of an assembly of particles in contact. Proc: Roy. Soc. A, vol. 269, p. 500-527, 1962.

Sander M. - Pratique de la mesure d'état de surface. Feinprûf Perthen $\mathrm{GmbH}$. 1992

Skinner A.E. - A note on the influence of interparticle friction on the shearing strengh of a random assembly of sphe. rical particles. Geotechnique, 19, $n^{\circ} 1$. p. 150-157, 1969.

Tschebotarioff G.P., Welch J.D. - Lateral earth pressure and friction between soil minerals. Proc., 2nd ICSMFE, Rotterdam, vol. 7, p. 135-138, 1948.

Wadell H.A. - Sphericity and roundness of rock particles. Am. I. Geol., vol. 41. p. 310-331, 1933.

Wilkins J.K. - A theory for the shear strenght of rockfill. Rock mechanics vol.2. p. 205-222, 1970. 